\title{
Hybrid Handshake MAC Protocol Based on Priority
}

\author{
CuiYan \\ Liberation Army of 95923 troops \\ Beijing, China \\ e-mail:625326139@qq.com
}

\author{
Xue Zhifeng \\ Communication and Information System \\ Northeastern University \\ Shenyang, China \\ e-mail: 625326139@qq.com
}

\author{
LiShaohua \\ Communication Institute, Equipment Academy of Air \\ Force \\ Beijing,China \\ e-mail: 717020833@qq.com
}

\author{
LiShengli \\ Shenyang Selection Center of Air Force Pilot Bureau \\ Shenyang,China \\ e-mail: 717020833@qq.com
}

\begin{abstract}
Aiming system of mobile phone the inner-flow competition problem, based on the research of IEEE 802.11 DCF protocol mixed handshake provide priority to network coding of continuous forward MAC protocol is proposed, providing access to a higher priority encoding condition data and ensuring the node delay controllable low priority, improve the real-time performance of the system. At the same time with the NS2 network simulation software to mixed into intra flow contention problem based on holding mobile phone system MAC protocol and the traditional competitive flow within the 802.11 MAC protocol simulation, And send the packet success rate and average delay requirement of service to end delay and the average network throughput are analyzed and compared, the intra flow contention problem based on introducing hybrid holding mobile phone system MAC protocol makes the network transmission increases, average end-to-end delay decreases the success rate, Improves network average throughput, optimize the network performance, To provide a guarantee for the real-time performance of the whole network system.
\end{abstract}

Keywords-802.11MAC; the inner-flow competition; priority; mixed handshake; NS-2

\section{INTRODUCTION}

Due to the rapid development of wireless communication terminal count and performance, Ad Hoc network applications in the civilian environment has also been a rapid development, if you want to interact in a non- temporary data communications infrastructure areas , you can easily set up a temporary Ad Hoc through network to achieve. Due to the inherent characteristics of Ad Hoc network, its applications with a common communication network has a very significant difference. Ad Hoc network is not easy to apply to multiple or no ability to pre-laying communications network infrastructure or communications network rapidly urgent need of scenarios, such as post-disaster emergency communications. From the development studies, in recent years, its applications are: military communications,emergency and emergency communications, wireless personal and home communications, wireless mesh
(Mesh Network), IOT (Internet of things) and sensor networks (wireless sensor Network, wsn). Ad Hoc network also has other advantages, there are many, which requires further exploration and study its applications.

However, due to the Ad hoc networks underlying communication technology is the wireless transmission used, therefore, compared with the cable channel, the channel bandwidth is much lower, and because it uses a wireless shared channel, the channel competition arising from conflicts and signal poor multipath fading, noise, interference and other factors make the Ad hoc networks ignore the channel quality, large node -end delay, delay jitter is too obvious. In the actual communication node achieved bandwidth is much smaller than the theoretical maximum bandwidth , and varies with time.

Mobile Ad Hoc network provides a distributed media competition based access control mechanism (DCF). In DCF mode, the carrier sensing mechanism to determine the state of the channel through the physical carrier sensin $\mathrm{g}$ and virtual carrier sensing.For channel reservation in ad dition using the RTS/CTS ${ }^{[1]}$ mechanism, the receiver send $\mathrm{s}$ an acknowledgement to the sender at the correct frame ACK received data frame DATA. Based on IEEE802 11 DCF, aiming for the competition in mobile phone the mixedholding mobile phone system, presents a hybrid handshake provide priority to network coding of continuous $\mathrm{f}$ orward $\mathrm{MAC}^{[2]}$ protocol. According to the data of priority, to adjust the contention window, so that the different priority data using different contention window to reduce the collision probability, channel, shorten transmission delay, improve the performance of the network.

\section{THE INNER-FLOW COMPETITION PROBLEM}

The inner-flow competition refers to the same information transmission path, the neighbor nodes within two hops in order to complete the transmission of information between the competing channel phenomenon. 


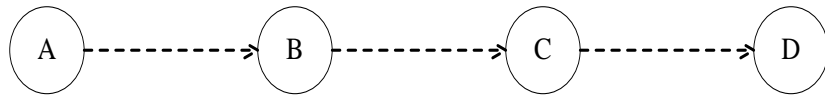

Figure 2.1 The inner-flow competition network topology

As shown in Figure 2.1, assume node A to send continuous data packet to the node $\mathrm{D}$, because the $\mathrm{D}$ in the communication range of $\mathrm{A}$, so to be forwarded by intermediate nodes in $\mathrm{B}$ and $\mathrm{C}$. At the same time, the node $\mathrm{A}$ data remains to be sent to $\mathrm{B}$, then the node $\mathrm{A}$ and B will be competing for the shared channel. Similarly, when a node $\mathrm{C}$ receives data frame node $\mathrm{B}$ forwarding, also need to forward data to the destination node $\mathrm{D}$, it will compete with the node of the $\mathrm{B}$ channel, when the communication power is larger even at A node channel competition. In accordance with this channel allocation mechanism of traditional $\mathrm{DCF}^{[3]}$, when entering the back off stage of channel contention access, each frame of data must pass through several times of waiting and retreat to reach the destination node. This is especially for the hop number, network in large scale network, will cause great delay, so that the decline of the network performance.So this paper introduces the mixed holding mobile phone for MAC protocol.

\section{A NODE PRIORITY}

The node data transmission in the network by setting different priorities, The Node is more important, the higher the priority, and all the nodes in the same priority constitute one and the same priority ${ }^{[4]}$ node set.

In order to ensure the fairness of network coding, the MAC protocol will be the time frame is divided into a plurality of time slots, where each time slot includes node initiates the propagation delay and channel for delay, media detection response delay. In addition, set every MAC time frame length $\mathrm{T}$, and divided into channel request total time $T_{1}$ and total time $T_{2}$ of data transmission.Considering the different node priority, the total time $T_{2}$ of data transmission is divided into different time periods, $t_{1}, t_{i}, \ldots, t_{n}$. The total time of each node to transmit data to the corresponding time slot $t_{i}$ contained. Frame structure as shown in Figure 3.1 protocol.The distribution of each node of the data transmission time is determined by type (3.1), the total number of nodes in which $\mathrm{m}$ this time frame in the process of channel competition $^{[5]}$.

$$
t_{i}=\frac{p_{i}}{\sum_{m} p_{i}} T_{2}
$$

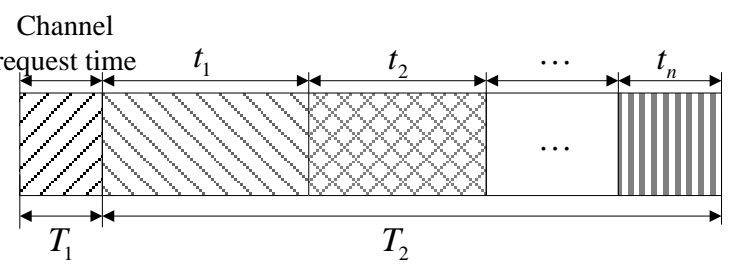

Figure 3.1 The frame structure of MAC protocol

\section{ACCESS PROCESS DESCRIPTION}

The basis of the traditional $802.11 \mathrm{DCF}$ protocol is improved, the forwarding mixed handshake priority based on MAC protocol, this protocol by ACK listen to the neighbor node to trigger the CTS handshake. In the node sends CTS message to the next hop node priority information node i (Priority Message) sent along with the message, send RTS and ACK messages are the priority information two hop node and with the message sent to the next hop node, after receiving the reply contains a node priority information one hop CTS. When ACK+ + message data representing the downstream node receives the upstream node receiving successful, downstream neighbor nodes located in short backoff directly after the CTS+ request is sent upstream nodes transmit data.

assume the source node A sends data to the destination D. Node A will according to the neighbor node priority information $P M_{A}+P M_{A+1}$ routing information in the additional transmitting to node $B$ in RTS, node B receives the RTS, see themselves in the A nodes are given priority information, is to maintain a short time slot IFS, where in IFS is formula (4.1) determination $^{[6]}$ :

$$
\text { IFS }_{i}=\left(1-P_{i}\right) * S I F S
$$

Due to the encoding data node contains package more, its priority is higher, then IFS is short, thus have more opportunities to channel competition.

When a B node IFS overtime not detected CTS messages to other nodes, the expected node information next hop is added to the CTS data frame sent together with. After the node A receives from the node B to start sending data to the CTS DATA.

The node $\mathrm{C}$ can monitor the arrival of node $\mathrm{B}$ sends to the A CTS, the next hop node contains information extracted from the $\mathrm{C}$, if the node in the node, $\mathrm{C}$ node $\mathrm{B}$ expected next hop node, the node $\mathrm{C} \mathrm{ACK}++$ signal monitoring node of $\mathrm{B}$, among them and by packet articles belt. When a node $\mathrm{C}$ monitor node $\mathrm{B} \mathrm{ACK}++$ signals, set the IFS, send CTS+ after a timeout. To the DATA sent to success D in turn .

This agreement in the $\mathrm{ACK}++$ is equivalent to $\mathrm{DCF}$ in the RTS protocol to send, will trigger the next hop node CTS, the node set the timer RTS after sending $\mathrm{ACK}++$, if RTS timeout has not yet received the next hop node CTS+, then restart $\mathrm{S}$ to shake hands, to send RTS $^{[7]}$. 


\section{MONITORING MECHANISM}

The protocol uses two hop neighbor nodes monitoring mechanism, can also listen to from the upstream node CTS and ACK virtual frame, which launched a channel request and allocation process.

(1) through the CTS virtual frame listener upstream nodes, selective withdrawal and monitor extraction information of neighbor nodes, can effectively avoid the CTS handshake conflict.

(2) through the ACK virtual frame listener upstream node two, extracting information of neighbor nodes, an aspect of the node can send the CTS, on the other hand, it can avoid the CTS conflict ${ }^{[8]}$.

In addition, the source node and the nodes start recovery timer RTS after sending ACK, the timer timeout if not yet received the downstream node reply CTS, shows that CTS or ACK is missing, it will immediately restart the RTS handshake ${ }^{[9]}$.

\section{THE SEND WINDOW ADJUSTMENT}

When sending failed, DCF uses BEB back off algorithm for all packets back off counter value COUNTER multiplied by 2 . Hybrid handshake improved continuous transfer of MAC protocol, the nodes with no different priority, with the formula (6.1) adjustment ${ }^{[10]}$ :

$$
C W_{\text {new }}=\min \left\{\left(C W_{\text {old }}+1\right) *\left(1-p_{i}\right)-1, C W_{\max }\right\}
$$

The algorithm will back off window according to the priority to dynamically adjust the node, compared with DCF back off algorithm for BEB, not simply fixed length increases or decreases, so as to ensure the high priority packets with smaller back off window after the collision, which can seize the channel faster, but also will not cause nodes back off value is too large ${ }^{[11]}$.

\section{SIMULATION AND PERFORMANCE ANALYSIS}

For comparison, the paper MAC layer using 802.11DCF, routing protocols AODV, handshake mechanism for hybrid MAC protocol based on the current competition and the introduction of simulation. Which defines each indicator simulation as follows:

(1)sending the message success rate.reflects the channel access real-time service node success rate.

(2) end to end delay. This system is used to measure the time delay indicator is defined as the issuing of a data packet to the destination node successfully receives the packet from the source node time. End delay is calculated as the average shown in Equation 7.1:

$$
\tau_{\text {end-to-end }}=\sum_{j=1}^{n} \sum_{i=1}^{m} \frac{1}{m \sqsubset n}\left(d_{i j}-s_{i j}\right)
$$

Where in said end delay average, $\mathrm{n}$ is the number of receiving nodes, $\mathrm{m}$ is the number of packets for all the ith destination node successfully received, is the i-th packet to the destination node $\mathrm{j}$ success time to reach the destination node was successful $\mathrm{j}$ i-th packet sent at the moment.

(3) The average network throughput. Is the ratio of the average of the sum of all the data packets per unit time at the receiving node successfully received all packets with the sum of the average of the source node sends. Generally, the lower the throughput, the greater the cost of its route described, but the throughput of the network is not necessarily smaller end delay will be higher, due to the end to end delay calculation is only sent successfully statistical data packets caused.

Using NS2 network simulation software, the simulation time is $600 \mathrm{~s}$, the simulation of each node in the data flow generation probability of Poisson distribution, topology of 20 nodes randomly distributed in a square area of the $1000 \times 1000 \mathrm{~m}$, the node communication radius $250 \mathrm{~m}$, bandwidth $2 \mathrm{M}$, using the AODV routing protocol, packet arrival time interval is 0.2 , and the introduction of the inner-flow competition problem based on hybrid holding mobile phone system MAC protocol simulation, sending the message success rate and average delay requirement of service to end delay and the average network throughput are analyzed, the packet transmission success rate reflects the channel access real-time service node success rate, average delay requirement of service to end delay reflect the quality of network service ${ }^{[12]}$. The simulation results in Figure 7.1, in figure 7.2,in figure 7.3.

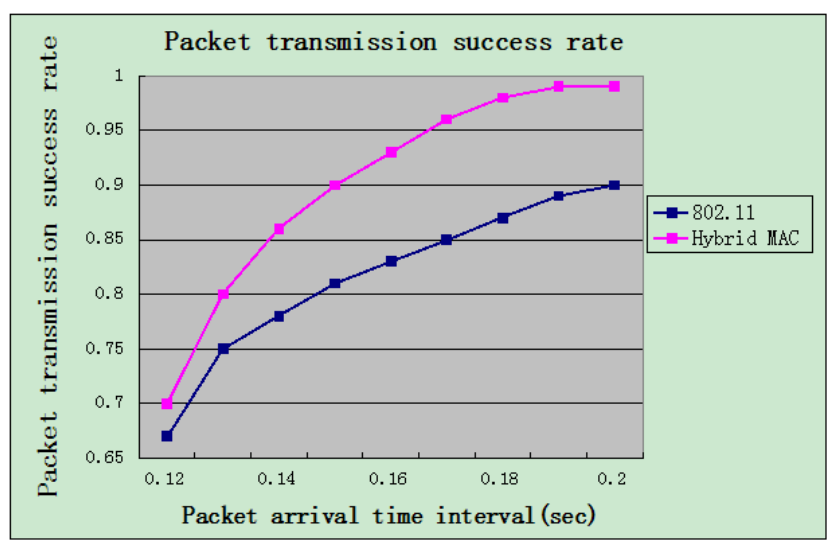

Figure7.1 packet delivery success rate

As shown in Figure 7.1, when the packet arrival time interval is 0.2 , message transmission priority mixed holding mobile phone system based on MAC protocol success rate was significantly higher than that of a competition problem within the stream 802.11 MAC protocol. 


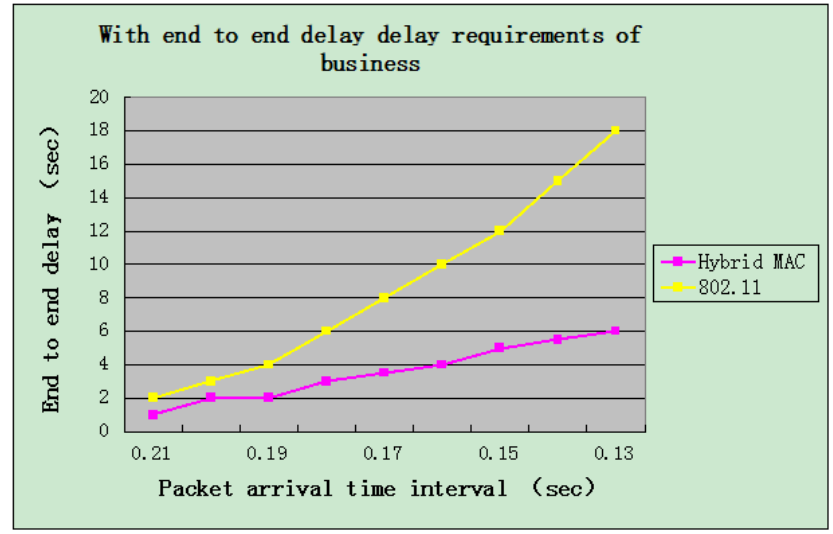

Figure 7.2 with end to end delay requirements of business

A delay in the business of end to end delay simulation results as shown in Figure 7.2, when traffic hours, end to end delay protocol are very small; when the business volume super saturation when the protocol, 802.11MAC protocol but as the service grows end to end delay increases gradually, the improved algorithm priority based on mixed mobile phone system holding MAC endto-end delay is always very small.

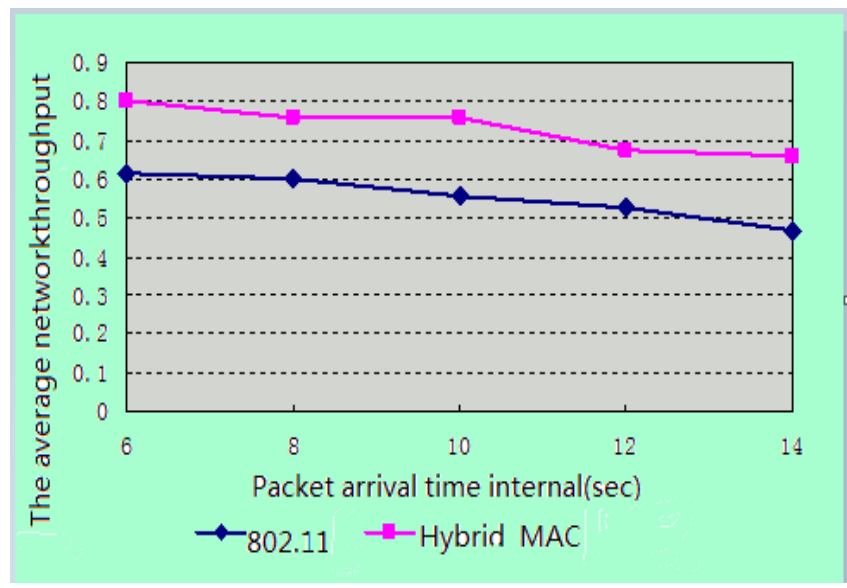

Figure 7.3 The average network throughput

The average network throughput simulation results as shown in Figure 7.3, the average throughput of networkbased mixed-priority MAC protocol handshake mechanism in the network transmission process flow was significantly higher than the competition issues have 802.11 MAC protocol.

\section{CONCLUSIONS}

Aiming the problem of mobile phone competition within a stream of the mixed holding mobile phone system, based on the research of IEEE 802.11 DCF protocol mixed handshake provide priority to network coding of continuous forward MAC protocol is proposed, which has the encoding condition data provides access to higher priority and ensure the node delay controllable low priority, to provide a guarantee for the real-time performance of the whole network system.

\section{REFERENCES}

[1] Tetsuya Shigeyasu, Daishi Inoue, Hiroshi Matsuno, Norihiko Morinaga.A new MAC protocol for reducing effect of needless transmission deferment induced by missed RTS/CTS handshake. Mobile Information Systems, 2009, Vol.5 (), pp.

[2] Jang-Ping Sheu, Chi-Hsun Liu, Shih-Lin Wu , Yu-Chee Tseng.A Priority MAC Protocol to Support Real-Time Traffic in Ad Hoc Networks.Wireless Networks , 2004, Vol.10 (1), pp.61-69.

[3] Zhenfeng Song.An adaptive cooperative MAC protocol compatible with legacy 802.11 DCF.Frontiers of Electrical and Electronic Engineering in China, 2010, Vol.5 (2), pp.197-202.

[4] Shih-Lin Wu, Jang-Ping Sheu, Chung-Chao Lee. A Fully Distributed MultiChannel MAC Protocol for Ad Hoc Networks Based on Location Information.International Journal of Pervasive Computing and Communications, 2007, Vol.2 (4), pp.

[5] Kaixin Xu, Mario Gerla, Sang Bae.Effectiveness of RTS/CTS handshake in IEEE 802.11 based ad hoc networks.Ad Hoc Networks , 2003, Vol.1 (1), pp.107-123.

[6] Ho T, Karger D, Medard M, et al. The benefits of coding over routing in a randomized setting[C]. IEEE International Symposium on Information Theory, Yokohama, Japan. 442, June,2003.

[7] Ho T, Medard M, Shi J, et al. On randomized network coding[C]. In: Proceedings of 41st Annual Allerton Conference on Communication, Control, and Computing, October 2003.

[8] Chou P A, Wu Y, Jain K. Practical network coding[C]. Allerton Conference on Communication, Control, and Computing,Monticello, IL, October 20, 2003.

[9] S. Lee and C. Kim, "Neighbor supporting Ad Hoc multicast routing protocol" in Proceedings of the ACM International Symposium on Mobile Ad Hoc Networking and Computing (Mobi Hoc), August 2000

[10] Wang Xiaodong, Huo Guangcheng, Sun Haiyan, et al. An Opportunistic routing for MANET based on partial network coding[J].Acta Electronica Sinica, 2010, 38(8): 1736-1740.

[11] S E Rouayheb, A Sprintson, C Georghiades. On the indes coding problem and its relation to network coding and matroid theory[J]. IEEE Transactions on Information Theory,2010,56(7):3187-3195.

[12] Hulya Seferoglu, Athina Markopoulou, "Delay-optimized network coding for video streaming over wireless networks"Communication (ICC), 20101EEElnternational Conference on, pp.1-5, 2010. 